\title{
Caenorhabditis elegans glia modulate neuronal activity and behavior
}

\author{
Randy F. Stout Jr. ${ }^{1}{ }^{*}$, Alexei Verkhratsky ${ }^{2,3,4}$ and Vladimir Parpura ${ }^{5,6 *}$ \\ 1 Department of Neuroscience, Albert Einstein College of Medicine, Bronx, NY, USA \\ ${ }^{2}$ Faculty of Life Sciences, The University of Manchester, Manchester, UK \\ ${ }^{3}$ IKERBASQUE, Basque Foundation for Science, Bilbao, Spain \\ ${ }^{4}$ Department of Neurosciences, University of the Basque Country UPV/EHU, Leioa, Spain \\ ${ }^{5}$ Department of Neurobiology, Center for Glial Biology in Medicine, Civitan International Research Center, Atomic Force Microscopy and Nanotechnology \\ Laboratories, and Evelyn F. McKnight Brain Institute, University of Alabama, Birmingham, AL, USA \\ ${ }^{6}$ Department of Biotechnology, University of Rijeka, Rijeka, Croatia
}

\section{Edited by:}

Tycho M. Hoogland, Netherlands

Institute for Neuroscience,

Netherlands

Reviewed by:

Bernd Kuhn, Okinawa Institute of Science and Technology Graduate University, Japan

Ping Liu, University of Connecticut Health Center, USA

*Correspondence:

Randy F. Stout Jr., The Dominick P. Purpura Department of Neuroscience, Rose F. Kennedy Center, Albert Einstein College of Medicine, 1410 Pelham Parkway South, Room 840, Bronx, NY 10461, USA

e-mail: randy.stout@einstein.yu.edu Vladimir Parpura, Department of Neurobiology, University of Alabama 1719 6th Avenue South, CIRC 429, Birmingham, AL 35242, USA

e-mail:vlad@uab.edu
Glial cells of Caenorhabditis elegans can modulate neuronal activity and behavior, which is the focus of this review. Initially, we provide an overview of neuroglial evolution, making a comparison between $C$. elegans glia and their genealogical counterparts. What follows is a brief discussion on $C$. elegans glia characteristics in terms of their exact numbers, germ layers origin, their necessity for proper development of sensory organs, and lack of their need for neuronal survival. The more specific roles that various glial cells have on neuron-based activity/behavior are succinctly presented. The cephalic sheath glia are important for development, maintenance and activity of central synapses, whereas the amphid glia seem to set the tone of sensory synapses; these glial cell types are ectoderm-derived. Mesoderm-derived Glial-Like cells in the nerve Ring (GLRs) appear to be a part of the circuit for production of motor movement of the worm anterior. Finally, we discuss tools and approaches utilized in studying C. elegans glia, which are assets available for this animal, making it an appealing model, not only in neurosciences, but in biology in general.

Keywords: glia, evolution, behavior, invertebrate, Caenorhabditis elegans

\section{INTRODUCTION: A BRIEF ON EVOLUTION OF NEUROGLIA}

"Nothing in biology makes sense except in the light of evolution". Theodosius Dobzhansky (1900-1975)

Evolution of the nervous system proceeded through an increase in number and complexity of the nervous elements and through their specialization into electrically excitable neurons connected through defined synaptic contacts and electrically non-excitable neuroglia forming networks through intercellular gap junctions. Intercellular chemical neurotransmission is, however, characteristic for both forms of the neural cells that express appropriate receptors and are capable of secreting neurotransmitters. The evolution of the nervous system was not a straight journey from less complex and accomplished networks to the more refined ones; at the turning point between invertebrates and vertebrates, a fundamental metamorphosis occurred that changed the overall structure of the central nervous system (CNS). This change is associated with an appearance of radial glia, that in the vertebrates, serve as a universal neural precursor and a guide for neural cells to migrate through the thickness of the neural tube thus creating a layered organization of the CNS (Kriegstein and Alvarez-Buylla, 2009). This layered organization is at odds with the CNS of invertebrates that essentially appears as fused neural ganglia. This major metamorphosis in the CNS organization also coincided with an extinction of the whole class of cells highly elaborated in the invertebrates - the parenchymal neuroglia. Indeed, in hemichordates (that barely have any CNS) glial cells seem to be completely absent, whereas in the primitive vertebrates (such as, for example, zebra fish or certain sharks and rays) the CNS contains essentially one functional layer that could be completely penetrated by radial glial cells, which perform major homeostatic functions. Increase in the thickness and size of the CNS strained the radial glia, and a new wave of evolution of parenchymal neuroglia began in some Elasmobranchi with "elaborated" brain (Reichenbach et al., 1987; Ari and Kálmán, 2008). This evolution rapidly resulted in high diversification of neuroglia, which assumed all major homeostatic and many defensive responsibilities in the mammalian brain. However, the direction of neuroglial evolution in Chordata is rather similar to that in the invertebrates, in which neuroglia similarly underwent remarkable morphological and 
function diversification while climbing the phylogenetic ladder from the most primitive bilateralia to the arthropods with their well-developed nervous system (for detailed account on glial evolution and references see Hartline, 2011; Verkhratsky and Butt, 2013).

The early evolutionary history of neuroglia is complex and is far from being characterized in detail. There is a general agreement that supportive neural cells are absent in the diffuse nervous system of Cnidaria and Ctenophora, although there are unconfirmed reports about the existence of glia-like cells in the ganglia of scyphomedusae (Bullock and Horridge, 1965). Supportive neural (glia-like) cells are present in the nervous system of Acoelomorpha that are generally considered as the first bilateralia (Bery et al., 2010). Glial cells are found in Nematoda (Heiman and Shaham, 2007), but are absent in phylogenetically more advanced Bryozoa and Gnathifera/Rotifera, even though the Rorifera have a proper CNS, in which neuronal structures are surrounded by either epithelial or muscle cells (Wallace and Smith, 2013). Nonetheless, in Annelida and Arthropoda the neuroglia are well defined and diversified; glial cells become responsible for homeostasis of the nervous system, they provide the hemolymph-brain barrier, they are capable of mounting astrogliotic response to insult and they create ancestral myelin-like sheath around axons (Deitmer et al., 1999; Edwards and Meinertzhagen, 2010). Here, we focus on recent research on the neuroglia of the nematode, Caenorhabditis elegans, with particular attention to reports of glia modifying neuronal activity and behavior of this round worm, along with discussion of advantages and limitations of studying glia in the worm, particularly for neuron-glial interactions outside of development.

\section{CHARACTERISTICS OF C. ELEGANS GLIA}

We provide only a brief overview of the general properties of C. elegans glia, as the detailed information can be found in recent reviews in respect to roles in development (Shaham, 2005, 2006; Oikonomou and Shaham, 2011) and evolutionary aspects of worm glia (Heiman and Shaham, 2007).

\section{THE EXACT NUMBER OF GLIA IN THE WORM}

Early studies of the nervous system of $C$. elegans produced extremely detailed and meticulously categorized structural information allowing identification of each neural cell (Ward et al., 1975; White et al., 1986; Hall and Russell, 1991). This makes C. elegans one of the few animals whose full complement of individual cells has been mapped throughout development and the only such animal widely used as a model in neuroscience. The neuroglia of $C$. elegans were described based on light and electron microscopy, i.e., (ultra)structural characteristics of these cells; they appear to be a part of the nervous system, but did not have morphological characteristics of neurons, i.e., lack pre-synaptic structures (Ward et al., 1975; Thomas, 1994). At that time, a set of 56 cells were classified as glia-like support cells in hermaphrodites (Altun and Hall, 2010). Developmental lineage maps further supported this classification; 50 of the 56 glia-like cells were shown to be of the ectodermal lineage. Of note, the "Handbook of Worm Anatomy" section of the website www.wormatlas.org provides an overview of the anatomic and developmental characteristics of the glia of C. elegans in the "support cells" sub-section on the hermaphrodite nervous system.

\section{AN EPHEMERAL COMPARISON OF WORM AND MAMMALIAN GLIA}

It is tempting to compare the glia of the worm to those of animals possessing more complex nervous systems. For these comparisons to be accurate, it is important to keep in mind that the nervous system of the worm likely contains fewer cells and connections that are optimal for its ecological niche, and not because it did not have enough evolutionary time or flexibility to attain greater complexity. Worms are not in possession of genes homologous to encoding glial fibrillary acidic protein (GFAP), a marker of astrocytes in mammals (although many other invertebrates express GFAP). Clearly, the functions that mammalian astrocytes perform in controlling blood flow and in contributing to the blood-brain barrier will not be possible for worm glia. Indeed, these functions are not required in the worms due to the small body size and characteristics of the environment in which they live. It is currently unknown if other functions of mammalian glia such as $\mathrm{K}^{+}$clearance, and vesicular release and re-uptake of neurotransmitters are performed by glial cells of the roundworm.

Some genetic pathways for glial specification and development do seem to be shared between glia of mammals and those of $C$. elegans. The transcription factor LIN-26 was found to be required for glial cell development and ablation of the lin-26 gene may cause cells that would become sheath glia to take on some characteristics of neurons (Labouesse et al., 1994). This was the first in a series of genetic/developmental findings that should be considered when we think about how glia evolved in different species. For example, the $h l h-17$ gene promoter has been used as a marker for the cephalic (CEP) sensilla sheath (CEPsh) glia (McMiller and Johnson, 2005). The hlh-17 gene seems to be important for development, but not initial specification of the CEPsh glia (Yoshimura et al., 2008). The hlh-17 gene has homology to the mammalian regulator of glial development Olig2. Genes that regulate the dor$\mathrm{sal} /$ ventral patterning of OLIGodendrocyte lineage transrctiption factor 2 (OLIG2) expression in mammals share homology to those required for normal HLH-17 expression specifically in the dorsal CEPsh glia of the worm (Yoshimura et al., 2008 and as reviewed in Oikonomou and Shaham, 2011).

\section{WORM GLIA ARE UNNECESSARY FOR NEURONAL SURVIVAL}

Based on experiments using gene deletions and/or cell ablations of glial precursors during development, we do know that a major difference between nervous system of $C$. elegans and nervous system of more advanced animals is that glia are mostly not required for survival of neurons within the mature nervous system of the worm and also the worms are able to survive and reproduce without glia. Except for the notable exceptions discussed later in this review, the glia of the worm seem to function mainly in guiding development of sensory structures in the worm and then act as a barrier by ensheathing the sensory structures in the adult worm. We know a great deal about how genes modulate the development and activity of $C$. elegans neurons, and also the neural molecular components that are shared (and not shared) between the neurons of C. elegans, Drosophila, mice, and human. 

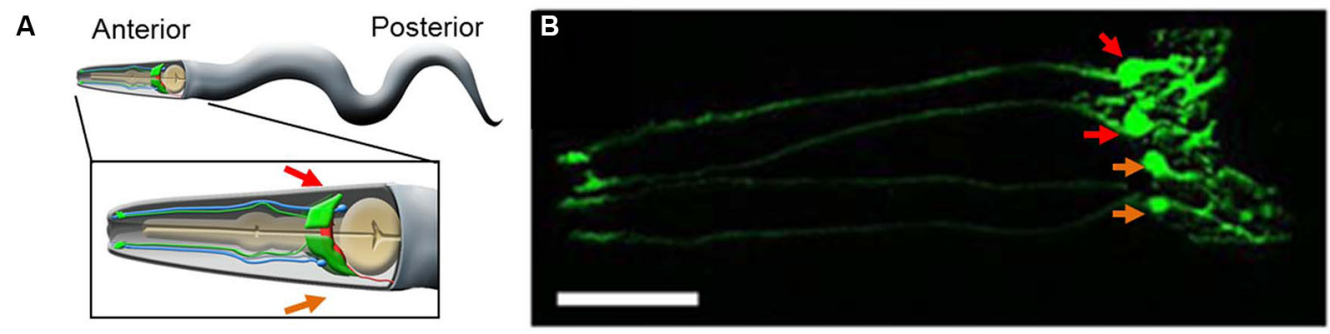

FIGURE 1 | The CEPsh glia. (A) A cartoon of an adult worm showing the four CEPsh glial cells (green) positioned in the anterior of the worm (inset). The CEPsh cell bodies with their velate extensions are positioned around the central nerve ring (red) which they enwrap along with the proximal section of the ventral nerve cord. Additionally, each CEPsh glial cell possesses a long anterior process, emanating to the anterior sensory tip, which closely interacts with the dendritic extension of a nearby CEP neuron (blue). Arrows indicate the dorsal (red arrows) and ventral (orange arrows) side of the worm. (B) A confocal image showing green fluorescent protein (GFP) expression driven by the h/h-17 promoter to visualize the four CEPsh glial cells (worm strain VPR839). The anterior (head) of a juvenile (larval stage 4) worm is shown; the worm is turned $\sim 45^{\circ}$ from "upright" such that all four CEP sheath cells are visible. The sheath portion of the cells that form a tube around the dendritic endings of the CEP neurons are seen at the left of the image. The dorsal (red arrows) and ventral (orange arrows) CEPsh cell bodies are seen. The thin sheet-like extensions that surround and invade the nerve ring are seen in the rightmost part of the image. Scale bar, $20 \mu \mathrm{m}$. Image adapted from Stout et al. (2013).
Examining the role of C. elegans glia in neural function may help us to understand of how these cells modulate neuronal activity or behavior in order to compare roles that glia of C. elegans and mammals play in information processing (see below).

\section{WORM GLIA PLAY A ROLE IN PROPER DEVELOPMENT OF SENSORY ORGANS}

Early ablation studies indicated that the sheath and socket glia played a role in the development of the ciliated sensory ending (Bargmann et al., 1990; Vowels and Thomas, 1994). The tractable genetics and other advantages of the worm were used to show that the glia of $C$. elegans affected sensory activity by controlling the development of cellular compartments surrounding sensory cilia (reviewed in Procko and Shaham, 2010). All of the ectodermderived glial cells of the worm are associated with the endings of these sensory neurons; neuronal dendritic endings together with glia form sensory organs of the worm known as sensilla. These specialized structures are prominent aspects of the nematode nervous system, and they fail to develop correctly without normal neuron-glia interactions. Neuronal development and maintenance of sensory structures require not only a set of genes expressed in the neurons, but also glial specific genes (reviewed in Oikonomou and Shaham, 2012). Attachments for sensory dendrites during migration of neurons during development also require factors released by the glia of $C$. elegans (Heiman and Shaham, 2009). Furthermore, it was recently suggested that the sensory synapse of the worm could be used as a model to study neuron-glia interactions in the human CNS (Shaham, 2010), albeit the usefulness of this model in this context remains to be seen.

Neuroglia in C. elegans perform at least four broad roles in the nervous system: (1) establishment of the location of neuronal structures; (2) regulation of sensory ending size and morphology; (3) a barrier that bundles and separates neuronal elements from other cells; and (4) modulation of neuronal activity. In a certain way this quartet resembles general roles that vertebrate glia are thought to have in the central and peripheral nervous systems.
The latter two roles, interrelated with some worm cells and behaviors, are further discussed below.

\section{THE CEP SHEATH GLIA IN SYNAPSE MAINTENANCE AND DOPAMINE-LINKED BEHAVIOR}

As already implicated, there appears to be a special class of cells among worm glia. We start our discussion by describing the four CEPsh glial cells (Figure 1). These ensheathing cells form a tubular structure surrounding the anterior tip of the sensory ending of CEP neurons and are therefore categorized into the group of 24 sheath glia found in the anterior of the worm. The CEPsh cells are unique in this glial pack in that they also extend thin sheet-like processes which ensheath the nerve ring, i.e., the worm "brain". Thin CEPsh cell processes also extend into the neuropil (White et al., 1986; Durbin, 1987; Oikonomou and Shaham, 2011).

There is morpho-functional heterogeneity between worm glia as in other animals. The morphology of the four CEPsh glia differs substantially from that of other sheath and socket glia (see below). The two ventral CEPsh cells express netrin (uncoordinated-6) but expression of this neuroligand involved in axon guidance is not detected or required in the dorsal pair of CEPsh glia (Hedgecock et al., 1990; Wadsworth et al., 1996; Yoshimura et al., 2008). Thin velate protrusions of the membrane of the CEPsh cells into the nerve ring appear to have some specificity as they are seen in proximity of the same neuronal synapses across different individual worms examined by electron microscopy (Ward et al., 1975; Durbin, 1987). Indeed, the CEPsh cells regulate synapse location through expression of the worm homolog of mammalian netrin (Colón-Ramos et al., 2007). More generally, the CEPsh glia are required for the maintenance of synaptic connectivity within the nerve ring (Shao et al., 2013, and reviewed in Yates, 2013).

Exciting, although circumstantial, evidence indicates that the CEPsh cells modulate dopamine-dependent behaviors in the worm, including feeding and a form of learning (Felton and Johnson, 2011). The hlh-17 gene encodes the basic Helix-LoopHelix transcription factor HLH-17 that is expressed almost 
A

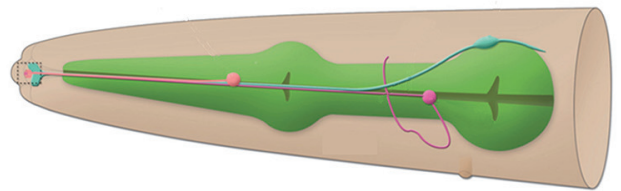

B
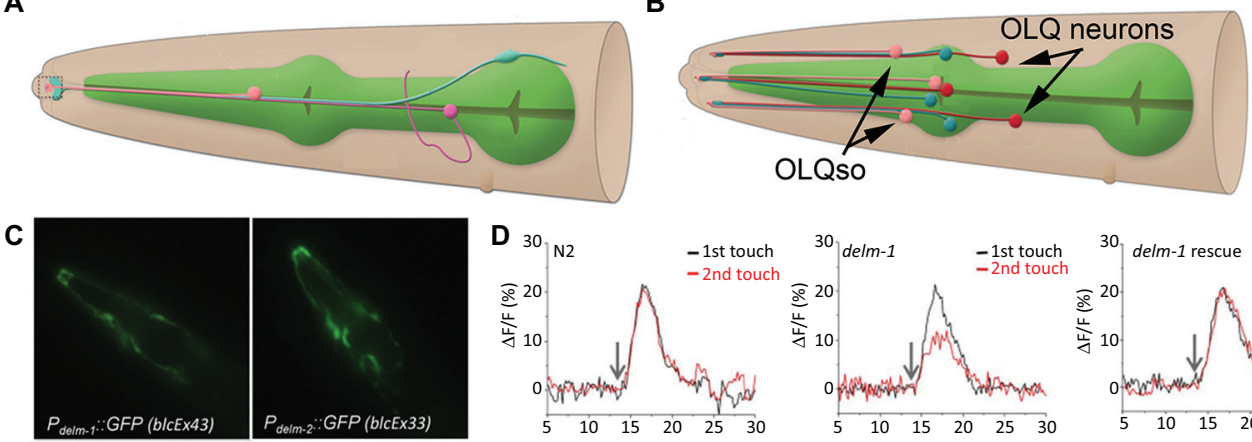

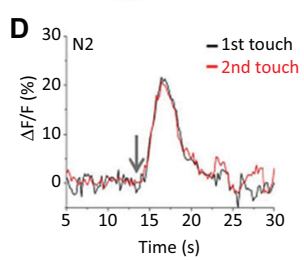

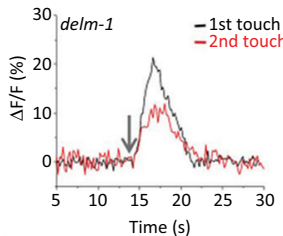

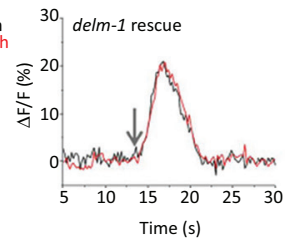

FIGURE 2 | The amphid sheath and outer labial sensilla socket cells. (A) The amphid sheath glia (blue) cell body is positioned near the nerve ring (not shown) and sends a long, thin process, along with a neuronal dendrite (magenta) and the amphid socket cell process (pink) to the anterior tip of the worm (left side, dashed box); adapted from wormatlas.org. (B) A cartoon showing the outer labial sensilla sheath (OLLsh and OLQsh, blue) and socket (OLLso and OLQso, pink) cells and their extensions to the anterior of the worm where they ensheath the ciliated dendrite of the neurons (OLL and OLQ, red); note that inner labial sensilla is not shown here; adapted from wormatlas.org. (C) The promoters for the delm-1 and delm-2 drive reporter GFP expression in the outer and inner labial sensilla socket glial cells (OLQso and ILso, respectively) of worms (strains blcEx43 and blcEx33, respectively). (D) Knock out of delm-1 leads to reduced OLQ neuron calcium response to mechanical stimulation of the worm (middle), while re-expression of the channel in OLQso glia (using the glial promoter itx-1) rescued the neuronal responsiveness (right); N2, background strain (left). C and D adapted from Han et al. (2013). exclusively in the CEPsh cells (McMiller and Johnson, 2005; Yoshimura et al., 2008). Disruption of the hlh-17 gene led to changes in egg-laying behavior, feeding behavior-plasticity deficits, and impaired a form of gustatory associative learning. The four CEPsh glia are closely associated with the four CEP neurons, which help mediate the aforementioned behaviors through release and up-take of dopamine (other neurons and neurotransmitters are mediators as well). Although the $h l h-17$ gene is not required for development/survival of the CEP neurons and sheath cells, the gene is required for dopamine-dependent behaviors as the loss of hlh-17 somehow affects dopamine signaling between the CEP neurons. These data represent an exciting hint that CEPsh glia modulate dopamine signaling and future research into this area of worm neurobiology is highly anticipated, especially in light of the role for dopaminergic signaling and dopamine transporters in human neurological diseases.

\section{AMPHID SHEATH AND SOCKET GLIA TUNE SENSORY NEURON ACTIVITY AND SENSORY BEHAVIOR}

There is strong evidence that channel activity within other (than CEPsh) sensilla-associated glia modulates neuronal activity, which in turn, affects behavioral responses to environmental stimuli (Wang et al., 2008, 2012; Han et al., 2013). These glia form sheaths around bundles of ciliated sensory dendrites (known collectively as the amphid inner and outer labial sensory organs) at the anterior tip of the worm. Promoter-reporter approaches mapped expression of several DEGenerin/Epithelial $\mathrm{Na}^{+}$Channels $(\mathrm{DEG} / \mathrm{ENaC})$ class channels to the sheath and socket glia. Through glial specific re-expression of DEG/ENaC channels ACid-sensitive Degenerin (ACD)-1 and DEgenerin Linked to Mechanosensation (DELM)-1,2 it was shown that expression of these channels in sheath and/or socket glia modulates the activity of sensory neurons (Wang et al., 2008, 2012; Han et al., 2013). The ACD-1 channel is required in the amphid sheath glia (depicted in Figure 2A), while the DELM-1 and 2 channels act in the inner and outer labial socket glia (depicted in Figure 2B). The DELM-1 and -2 are required in the glia but not in neurons in order for the worm to perform a set of foraging related behaviors. In the cells expressing the DELM-1 and 2 channels only re-expression under a glial socket cell promoter rescued sensory behavior and neuronal calcium signaling deficits (Figures 2C, D). Similarly the effects of acd-1 knockout were only rescued by re-expressing the gene in the amphid sheath glia.

So, how could expression of an ion channel in glia modulate sensory neuron activity and mechanosensory behavioral responses? It has been postulated that activity of glial DEG/ $\mathrm{ENaC}$ channels leads to an increase in extracellular $\mathrm{K}^{+}$and thereby to an increase in excitability of the nearby neuronal processes. There is some semblance of this mechanism to specialized regulation of $\mathrm{K}^{+}$concentration at sensory endings of vertebrates (Pacinian corpuscle), which modulates sensory neuron activity (Hyinsky et al., 1976). In another study, calcium responses of chemosensory neurons (Amphid Wing Cell (AWC) neurons) to chemical (isoamyl alcohol) stimulations near the detection threshold were used to show that loss of the glial specific ACD-1 channel is required for normal AWC neuron calcium response, but that ACD-1 is not directly affected by such stimulation. Artificial manipulation of the baseline activity of the AWC neurons by expressing a human capsaicin-sensitive TRPV1 channel in the worm's AWC neurons masked the effect of the loss of glial ACD-1. Finally, it was demonstrated that the ACD-1 channels were localized to the anterior end of the worm where the AWC sensory dendrites interacted with chemical stimulant. While all aspects of this neuron-glia interaction are not clear, available evidence supports the idea that the glia 

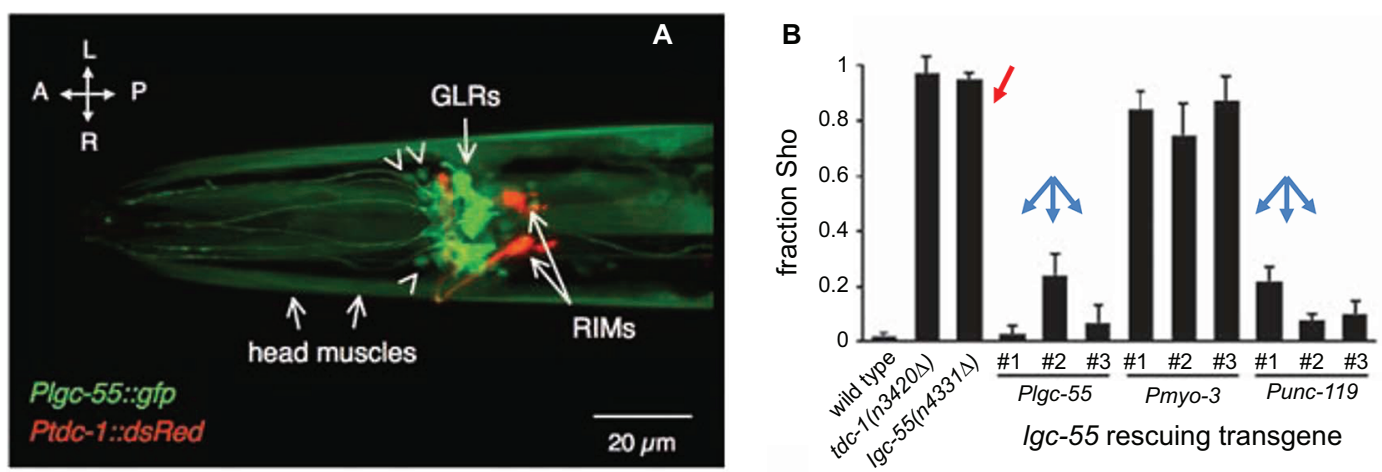

FIGURE 3 | LGC-55 expressed in the GLRs is required for normal tyraminergic modulation of head movements by $\boldsymbol{C}$. elegans. (A) The promoter for the tyramine receptor LGC-55 drives expression of GFP in the six GLRs and head muscle cells of $C$. elegans; RIMs, tyraminergic interneurons expressing red fluorescent protein. Orientation arrows, L-R, left-right, A-P, anterior-posterior. Arrowheads indicate some of the unidentified head neurons that express the gfp transgene. (B) Worms carrying a mutation in the lgc-55 gene (or in the $t d c$-1 gene, encoding tyrosine decarboxylase, an enzyme that converts L-tyrosine to tyramine) do not suppress foraging movements when crawling backward (Sho phenotype; red arrow). Re-expression of the LGC-55 driven by either the lgc-55 or unc-119 (pan-neural) promoters (blue arrows), but not by myo-3 (muscle) promoter, rescues the behavioral phenotype, indicating that glia or neurons are responsible for the behavior. Adapted from Ringstad et al. (2009). modulate the threshold for neuronal excitability. Furthermore, it seems that two different sets of glial cells modulate the activity of sensory synapses using different ion channels and that this action is specific to a subset of synapses within the same glial "cradle" structure, this latter being a concept put forward to characterize the role of astrocytes at the vertebrate synapse (Nedergaard and Verkhratsky, 2012). In conclusion, the CEPsh glia are important for development, maintenance and activity of central synapses, whereas the amphid glia seem to set the tone of sensory synapses.

\section{THE GLRS: AN UNORTHODOX GLIAL TYPE}

We next discuss the evidence for a signaling pathway between neurons to muscle cells that may pass through an unusual type of C. elegans glial cells. Namely, the six Glial-Like cells in the nerve Ring (GLRs) were named based on their morphology and location; however, unlike other worm glia the GLRs are of mesodermal origin (reviewed in www.wormatlas.org (Altun and Hall, 2009)). It is somewhat surprising that a cell type of different origin can so closely morphologically resemble all other glial cells of the worm. Because of the lineage, a comparison to mammalian microglia is tempting, especially in light of a GLR cell having been observed (by electron microscopy) apparently engulfing a dying CEP neuron (Nass et al., 2002). The GLRs are integrated into the nervous system of the worm and contribute to the development of the nerve ring and pharynx (muscle-based feeding organ of the worm). GLRs are connected to both neurons and muscle cells in the head by gap junctions and may be part of the circuit for producing specialized fine motor movements of anterior of the worm during foraging that are analogous to neck movements of vertebrates (White et al., 1986; Ringstad et al., 2009). Two reports examining the role of Ligand-Gated ion Channel (LGC)55 , a tyramine receptor, pointed to its expression in the GLRs. One report indicates that LGC-55 could function in GLRs or neurons to suppress foraging activity during backward movement
(Ringstad et al., 2009; Figure 3). Another report points to the function of the LGC-55 in muscle and to a lesser extent in neurons (Pirri et al., 2009).

Potential involvement of GLRs in a circuit relevant for behavior is intriguing and warrants further research. This research, into the function of GLRs, however, has been hampered by the lack of a cell specific promoter. This challenge could potentially be circumvented with combinatorial-conditional promoter systems to limit genetically based markers, activity indicators, or functional modulators to the GLRs (Voutev and Hubbard, 2008). The finding that there are gap junctions between the GLRs and neurons is intriguing especially in light of recent discoveries that gap junctions seem to connect the neurons to glia of the sensory ganglia (Suadicani et al., 2010) and as reviewed by Hanani (2012) and Huang et al. (2013) and in the developing CNS (Pakhotin and Verkhratsky, 2005) of mice. The protein subunits that make up the gap junction channels of invertebrates (innexins) and vertebrates (connexins) share no protein sequence homology, but form similar macromolecular structures that connect the cytoplasm of adjacent cells, as reviewed in Scemes et al. (2007) and Simonsen et al. (2014). Additionally, the GLRs of the worm have taken on morphological characteristics of glia although they follow different developmental path, and express a different set of genes than other C. elegans glia. This may represent a case of compound convergent evolution and points to a role of gap junction based neuron-glia interaction as a rare but fundamental process, since it seems to have arisen independently in highly divergent nervous systems. Therefore, this may be a particularly important area for future study. The genome of C. elegans contains 25 genes for innexins (Starich et al., 2001); a promoter-reporter based survey indicated that the sheath and socket glia also express several innexin genes (Altun et al., 2009). This is another common feature shared between the CEPsh glia and vertebrate astrocytesprominent expression of gap junctions. However, gap junctions 


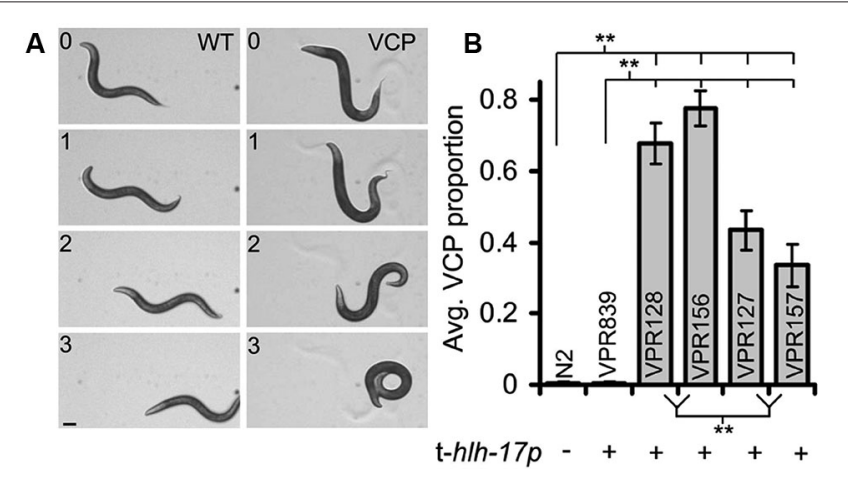

FIGURE 4 | A subset of $C$. elegans strains carrying transgenic arrays containing the trans-hlh-17 promoter (t-hlh-17p) to drive expression of reporters in the CEPsh glial cells display a ventral coiler phenotype (VCP) during backward movement. (A) A time-lapse montage of worms showing a normal/sinusoidal pattern during backwards locomotion (WT, left column). Worms with many copies of the t-h/h-17p coil when they attempt to crawl in reverse (VCP, right column; strain VPR156). Numbers indicate time in seconds. Scale bar, $100 \mu \mathrm{m}$. (B) Non-transgenic worms (WT, N2) do not display the VCP, but different strains of worms (VPR) containing the $\mathrm{t}-\mathrm{h} / \mathrm{h}-17 \mathrm{p}$, except the VPR839 strain, display varying degrees of abnormal locomotion. ** Indicates a significant difference. Adapted from Stout et al. (2013).

connecting CEPsh glia to other cells have not been investigated and this is currently a woefully understudied aspect of worm glia.

The recently discovered/renamed cell type known as telocytes may be the mammalian equivalent of the worm GLRs (Gherghiceanu and Popescu, 2011; Cretoiu et al., 2012; Smythies and Edelstein, 2014). If the set of genes expressed by the GLRs turns out to share similarity to the set expressed in telocytes, perhaps the GLR-type should be reclassified from glia to ancestral telocytes. Although this is currently purely speculative, such classification would establish the GLRs as a model to study the biology of this exciting new vertebrate cell type.

Finally, it is worth mentioning that the GLRs are connected by gap junctions to ring motor neurons which themselves receive synaptic input from cells that make up a gap junction-mediated circuit with coincidence detection features (Rabinowitch et al., 2013). It will be important to test if the activity of the GLRs influence this or associated circuits and if gap junctions are involved in GLR physiology.

\section{APPROACHES IN STUDYING GLIA IN C. ELEGANS}

Several themes emerge in the approaches used in the research highlighted above. Rescue of genetic ablation-induced changes to neuronal function or behavior through use of glia-specific promoters is widely used in both invertebrates and vertebrates. This approach is particularly powerful for C. elegans since an enormous number of mutant strains are available. The determined cell lineage and ease of specific cell identification is an advantage. Additionally, C. elegans is probably the easiest and cheapest animal model organism in which to produce and maintain transgenic animal lines. Cell-type specific promoters are available and, in combination with the phenomenon of transgene mosaicism, genetic rescue or other manipulations can be targeted to individual glial cells (Colón-Ramos et al., 2007; Yoshimura et al., 2008). Transgenes can affect neuronal and behavioral phenotypes in unexpected ways, but if such effects are detected they can be controlled for by using alternative strategies for transgene introduction (Kage-Nakadai et al., 2012; Stout et al., 2013). For example, worms carrying many (more than $\sim 200$ ) copies of the transgenic hlh-17 promoter display a ventral coiling behavior during backward locomotion (Figure 4); coiling occurrences correlated to the transgene promoter copy number (Stout et al., 2013). This behavior is variable across different transgenic worm strains and can range from subtle to near complete inability for worms to crawl in the backward direction. If worm strains used in studies on worm behavior exhibit even a very mild form of the ventral coiling behavior it would be expected to have a major impact on sensitive behavioral tests such as salt-food associative learning and measures of reversal frequency or on measures of shape of body-bending during locomotion. Future studies where behavioral alterations are attributed to glia will need to control for the possibility that promoters used to target glial cells may cause (in)direct behavioral effects (Stout et al., 2013).

Optical methods for recording neuronal activity are currently more widely used than electrophysiological methods due to difficulty in accessing neurons with patch electrodes which is hampered by the worm cuticle and internal pressure (Kerr et al., 2000; Kerr and Schafer, 2006). Thus far, glial cell activity has only been assessed by genetically encoded optical indicators (Stout and Parpura, 2011; Wang et al., 2012). The ability to culture embryonic and adult stage C. elegans cells (Christensen et al., 2002; Frøkjaer-Jensen et al., 2006; Strange et al., 2007), including glia (Stout and Parpura, 2012; Sangaletti and Bianchi, 2013), should ease electrophysiological access to glial cells and has allowed acute application of pharmacological manipulations (Stout and Parpura, 2011; Figure 5). For instance, the combination of genetically encoded indicators, mutant strains carrying deletions of voltage-gated $\mathrm{Ca}^{2+}$ channels (VGCCs), and pharmacological treatments showed that cultured CEPsh glia respond to membrane depolarization with increases in intracellular $\mathrm{Ca}^{2+}$ mediated by various types of VGCCs (e.g., the role of L-type is shown in Figure 5). Optogenetic manipulations, i.e., use of channelrhodopsin, have been very useful in the study of C. elegans neurons (Nagel et al., 2005), but behavioral effects of lightactivated channels have not been hitherto reported for C. elegans glia.

It may seem surprising that no reports of laser or genetic ablation of all CEPsh glia in adult worms have been published, particularly since post-embryonic, larval-stage ablation of a subset of the CEPsh cells led to interesting phenotypes. Briefly, when the precursor cells of the CEPsh glia are ablated during embryonic development the CEP neuron dendrites are shortened, axons in the nerve ring are disrupted, and the entire nerve ring is disrupted in some animals that lack the CEPsh glia. Some worms even failed to develop past the larval L1 stage when CEPsh glia were ablated (Yoshimura et al., 2008). Heat shock-inducible expression of a cell-killing caspase (Chelur and Chalfie, 2007) in adult CEPsh cells led to defects in synapse maintenance in the Amphid 

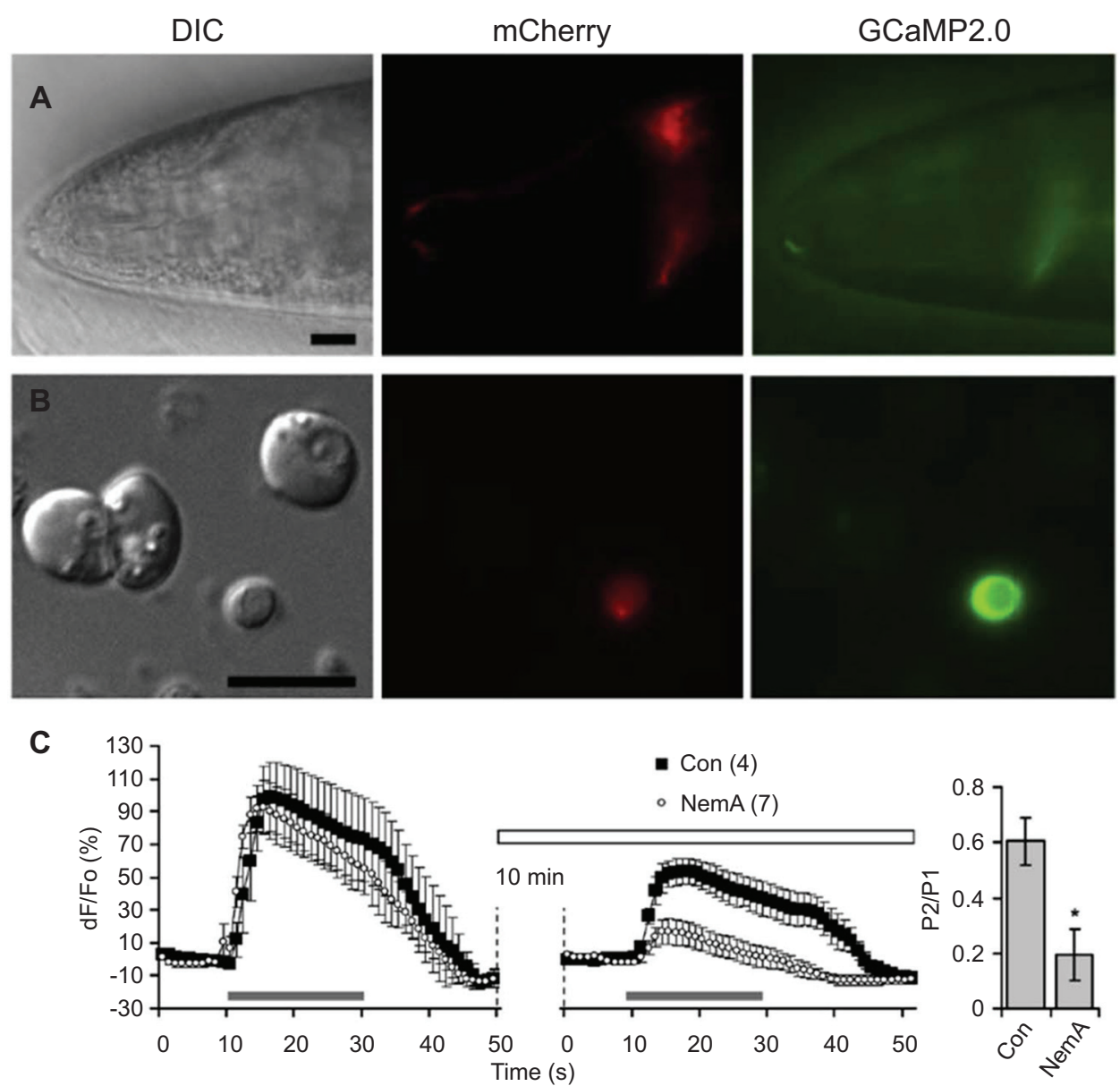

FIGURE 5 | L-type voltage-gated $\mathrm{Ca}^{2+}$ channels (VGCCs) play a role in depolarization-induced intracellular $\mathrm{Ca}^{2+}$ elevations in CEPsh glial cells. (A) The $h / h-17$ promoter can be used to drive expression of a red fluorescent protein marker (red, mCherry) in the CEPsh glia along with a fluorescent protein-based $\mathrm{Ca}^{2+}$ sensor (green, GCaMP2,0). Differential interference contrast (DIC). An anterior portion of an $L 4$ stage worm (VPR108 strain) is shown. (B) CEPsh glial cells in mixed culture prepared from embryos can be identified based on their mCherry/GCaMP2.0 expression. (C) Time-lapse of GCaMP2.0 fluorescence emission from
CEPsh glial cells. Paired-pulse application of a depolarization stimulus, high extracellular potassium (HiK+, $100 \mathrm{mM}$; horizontal grey bar) to CEPsh glial cells results in an elevation of intracellular $\mathrm{Ca}^{2+}$ levels (black squares). Nemadipine-A (NemA), a pharmacological L-type VGCC blocker, can be used to test the channels present in glial cells in culture (horizontal open bar); Con, sham stimulated control (right, bar graph). Ratio of the peak $\mathrm{Ca}^{2+}$ level in response to the second $\mathrm{HiK}^{+}$application (P2) over the first application (P1). * Indicates a significant difference. Adapted from Stout and Parpura (2011).
Interneuron Y (AIY), but incomplete ablation of all CEPsh glia across individual worms may have occurred (Shao et al., 2013). These data highlight the need for future research to further assess the effect of acute loss of CEPsh glia in adulthood. The present lack of experiments ablating the all CEPsh glia in adult worms, however, may be due to the unusual nature of the CEPsh glia in that they have a large cellular surface area that is spread over a large portion of the anterior nervous system.

In general, many of the methods that worked so well for the study of worm neurons have been harder to implement for glial biology. The development of new optogenetic probes and channels along with our rapidly increasing knowledge of gene expression in worm glia (Spencer et al., 2011) can be expected to make the discoveries we described in this review a start to an exciting time in research on glia-neuron interactions in the adult worm.

\section{ENVOI}

The intent of this focus review was to summarize current evidence indicating that the glia of $C$. elegans have an important role in modulation of neuronal activity and behavior. Indeed, future studies are needed to understand details of glial roles in the nervous system of this nematode. Meanwhile, C. elegans has proven enormously helpful in unveiling mysteries surrounding the operation of not only the nervous system, but also of many other basic biological functions. Consequently, it shall not come as a surprise that the National Institutes of Health list C. elegans as one of the model organisms for biomedical research. ${ }^{1}$ This "feather in the cap" has been earned as a consequence of $C$. elegans being one of the animals whose full complement of individual cells has been mapped throughout development and

\footnotetext{
${ }^{1}$ http://www.nih.gov/science/models/
} 
due to the ease of genetic manipulations, with rapid outcomes, which this nematode offers. Moreover, there are publicly available information resources (e.g., WormAtlas, WormBase and WormBook; http://www.wormatlas.org, http://www.wormbase.org, and http://www.wormbook.org, respectively), and consortia providing various mutation/knockout/transgenic strains on a thrifty budget (e.g., Caenorhabditis Genetics Center at University of Minnesota, Minneapolis, MN; http://www.cbs.umn.edu/research/resources/cgc), making $C$. elegans an appealing model in neurosciences and biomedicine.

\section{ACKNOWLEDGMENTS}

Randy F. Stout's research is supported by the National Institutes of Health (NIH) institutional training grant (5T32NS00743914) to the Dominick P. Purpura Department of Neuroscience. Other authors' research is supported by the Alzheimer's Research Trust (UK) Programme Grant (ART/PG2004A/1) to Alexei Verkhratsky; by the NIH (The Eunice Kennedy Shriver National Institute of Child Health and Human Development award HD078678) to Vladimir Parpura.

\section{REFERENCES}

Altun, Z. F., Chen, B., Wang, Z. W., and Hall, D. H. (2009). High resolution map of Caenorhabditis elegans gap junction proteins. Dev. Dyn. 238, 1936-1950. doi: $10.1002 /$ dvdy.22025

Altun, Z. F., and Hall, D. H. (2009). "Muscle system, GLR cells," in Worm Atlas. doi: 10.3908/wormatlas.1.9

Altun, Z. F., and Hall, D. H. (2010). "Nervous system, neuronal support cells," in Worm Atlas. doi: 10.3908/wormatlas.1.19

Ari, C., and Kálmán, M. (2008). Evolutionary changes of astroglia in Elasmobranchii comparing to amniotes: a study based on three immunohistochemical markers (GFAP, S-100, and glutamine synthetase). Brain Behav. Evol. 71, 305324. doi: 10.1159/000129654

Bargmann, C. I., Thomas, J. H., and Horvitz, H. R. (1990). Chemosensory cell function in the behavior and development of Caenorhabditis elegans. Cold Spring Harb. Symp. Quant. Biol. 55, 529-538. doi: 10.1101/sqb.1990.055.01.051

Bery, A., Cardona, A., Martinez, P., and Hartenstein, V. (2010). Structure of the central nervous system of a juvenile acoel, Symsagittifera roscoffensis. Dev. Genes Evol. 220, 61-76. doi: 10.1007/s00427-010-0328-2

Bullock, T. H., and Horridge, G. A. (1965). Structure and Function in the Nervous System of Invertebrates. (Vol. 2), San Francisco and London : W. H. Freeman a. Comp. Ltd., XXVIII + 1722 .

Chelur, D. S., and Chalfie, M. (2007). Targeted cell killing by reconstituted caspases. Proc. Natl. Acad. Sci. U S A 104, 2283-2288. doi: 10.1073/pnas.0610877104

Christensen, M., Estevez, A., Yin, X., Fox, R., Morrison, R., McDonnell, M., et al. (2002). A primary culture system for functional analysis of C. elegans neurons and muscle cells. Neuron 33, 503-514. doi: 10.1016/s0896-6273(02)00591-3

Colón-Ramos, D. A., Margeta, M. A., and Shen, K. (2007). Glia promote local synaptogenesis through UNC-6 (netrin) signaling in C. elegans. Science 318, 103-106. doi: 10.1126/science. 1143762

Cretoiu, D., Cretoiu, S. M., Simionescu, A. A., and Popescu, L. M. (2012). Telocytes, a distinct type of cell among the stromal cells present in the lamina propria of jejunum. Histol. Histopathol. 27, 1067-1078.

Deitmer, J. W., Rose, C. R., Munsch, T., Schmidt, J., Nett, W., Schneider, H.-P., et al. (1999). Leech giant glial cell: functional role. Glia 28, 175-182. doi: 10 . 1002/(sici)1098-1136(199912)28:3 < 175::aid-glial > 3.0.co;2-7

Durbin, R. M. (1987). Studies on the Development and Organisation of the Nervous System of Caenorhabditis Elegans. Ph.D thesis. UK: University of Cambridge.

Edwards, T. N., and Meinertzhagen, I. A. (2010). The functional organisation of glia in the adult brain of Drosophila and other insects. Prog. Neurobiol. 90, 471-497. doi: 10.1016/j.pneurobio.2010.01.001

Felton, C. M., and Johnson, C. M. (2011). Modulation of dopamine-dependent behaviors by the Caenorhabditis elegans Olig homolog HLH-17. J. Neurosci. Res. 89, 1627-1636. doi: 10.1002/jnr.22694
Frøkjaer-Jensen, C., Kindt, K. S., Kerr, R. A., Suzuki, H., Melnik-Martinez, K. Gerstbreih, B., et al. (2006). Effects of voltage-gated calcium channel subunit genes on calcium influx in cultured C. elegans mechanosensory neurons. $J$. Neurobiol. 66, 1125-1139. doi: 10.1002/neu.20261

Gherghiceanu, M., and Popescu, L. M. (2011). Heterocellular communication in the heart: electron tomography of telocyte-myocyte junctions. J. Cell. Mol. Med. 15, 1005-1011. doi: 10.1111/j.1582-4934.2011.01299.x

Hall, D. H., and Russell, R. L. (1991). The posterior nervous system of the nematode Caenorhabditis elegans: serial reconstruction of identified neurons and complete pattern of synaptic interactions. J. Neurosci. 11, 1-22.

Han, L., Wang, Y., Sangaletti, R., D’Urso, G., Lu, Y., Shaham, S., et al. (2013). Two novel DEG/ENaC channel subunits expressed in glia are needed for nosetouch sensitivity in Caenorhabditis elegans. J. Neurosci. 33, 936-949. doi: 10. 1523/jneurosci.2749-12.2013

Hanani, M. (2012). Intercellular communication in sensory ganglia by purinergic receptors and gap junctions: implications for chronic pain. Brain Res. 1487, 183 191. doi: 10.1016/j.brainres.2012.03.070

Hartline, D. K. (2011). The evolutionary origins of glia. Glia 59, 1215-1236. doi: 10. 1002/glia.21149

Hedgecock, E. M., Culotti, J. G., and Hall, D. H. (1990). The unc-5, unc-6 and unc40 genes guide circumferential migrations of pioneer axons and mesodermal cells on the epidermis in C. elegans. Neuron 4, 61-85. doi: 10.1016/08966273(90)90444-k

Heiman, M. G., and Shaham, S. (2007). Ancestral roles of glia suggested by the nervous system of Caenorhabditis elegans. Neuron Glia Biol. 3, 55-61. doi: 10. 1017/s1740925x07000609

Heiman, M. G., and Shaham, S. (2009). DEX-1 and DYF-7 establish sensory dendrite length by anchoring dendritic tips during cell migration. Cell 137, 344355. doi: 10.1016/j.cell.2009.01.057

Huang, L. Y. M., Gu, Y., and Chen, Y. (2013). Communication between neuronal somata and satellite glial cells in sensory ganglia. Glia 61, 1571-1581. doi: 10. 1002/glia.22541

Hyinsky, O., Akoev, G., Krasnikova, T., and Elman, S. (1976). K and Na ion content in the Pacinian corpuscle fluid and its role in the activity of receptors. Pflugers Arch. 361, 279-285. doi: 10.1007/bf00587293

Kage-Nakadai, E., Kobuna, H., Funatsu, O., Otori, M., Gengyo-Ando, K., Yoshina, S., et al. (2012). Single/low-copy integration of transgenes in Caenorhabditis elegans using an ultraviolet trimethylpsoralen method. BMC Biotechnol. 12:1. doi: 10.1186/1472-6750-12-1

Kerr, R., Lev-Ram, V., Baird, G., Vincent, P., Tsien, R. Y., and Schafer, W. R. (2000). Optical imaging of calcium transients in neurons and pharyngeal muscle of C. elegans. Neuron 26, 583-594. doi: 10.1016/s0896-6273(00)8 1196-4

Kerr, R. A., and Schafer, W. R. (2006). Intracellular Ca2+ imaging in C. elegans. Methods Mol. Biol. 351, 253-264. doi: 10.1385/1-59745-151-7:253

Kriegstein, A., and Alvarez-Buylla, A. (2009). The glial nature of embryonic and adult neural stem cells. Annu. Rev. Neurosci. 32, 149-184. doi: 10.1146/annurev. neuro.051508.135600

Labouesse, M., Sookhareea, S., and Horvitz, H. R. (1994). The Caenorhabditis elegans gene lin-26 is required to specify the fates of hypodermal cells and encodes a presumptive zinc-finger transcription factor. Development 120, 23592368.

McMiller, T. L., and Johnson, C. M. (2005). Molecular characterization of HLH-17, a C. elegans bHLH protein required for normal larval development. Gene 356, 1-10. doi: 10.1016/j.gene.2005.05.003

Nagel, G., Brauner, M., Liewald, J. F., Adeishvili, N., Bamberg, E., and Gottschalk, A. (2005). Light activation of channelrhodopsin-2 in excitable cells of Caenorhabditis elegans triggers rapid behavioral responses. Curr. Biol. 15, 2279-2284 doi: 10.1016/j.cub.2005.11.032

Nass, R., Hall, D. H., Miller, D. M. 3rd., and Blakely, R. D. (2002). Neurotoxininduced degeneration of dopamine neurons in Caenorhabditis elegans. Proc. Natl. Acad. Sci. U S A 99, 3264-3269. doi: 10.1073/pnas.042497999

Nedergaard, M., and Verkhratsky, A. (2012). Artifact versus reality-how astrocytes contribute to synaptic events. Glia 60, 1013-1023. doi: 10.1002/glia.22288

Oikonomou, G., and Shaham, S. (2011). The glia of Caenorhabditis elegans. Glia 59, 1253-1263. doi: 10.1002/glia.21084

Oikonomou, G., and Shaham, S. (2012). On the morphogenesis of glial compartments in the sensory organs of Caenorhabditis elegans. Worm 1, 51-55. doi: 10. 4161/worm. 19343 
Pakhotin, P., and Verkhratsky, A. (2005). Electrical synapses between Bergmann glial cells and Purkinje neurones in rat cerebellar slices. Mol. Cell. Neurosci. 28, 79-84. doi: 10.1016/j.mcn.2004.08.014

Pirri, J. K., McPherson, A. D., Donnelly, J. L., Francis, M. M., and Alkema, M. J. (2009). A tyramine-gated chloride channel coordinates distinct motor programs of a Caenorhabditis elegans escape response. Neuron 62, 526-538. doi: 10.1016/j. neuron.2009.04.013

Procko, C., and Shaham, S. (2010). Assisted morphogenesis: glial control of dendrite shapes. Curr. Opin. Cell Biol. 22, 560-565. doi: 10.1016/j.ceb.2010. 07.005

Rabinowitch, I., Chatzigeorgiou, M., and Schafer, W. R. (2013). A gap junction circuit enhances processing of coincident mechanosensory inputs. Curr. Biol. 23, 963-967. doi: 10.1016/j.cub.2013.04.030

Reichenbach, A., Neumann, M., and Bruckner, G. (1987). Cell length to diameter relation of rat fetal radial glia-does impaired $\mathrm{K}+$ transport capacity of long thin cells cause their perinatal transformation into multipolar astrocytes? Neurosci. Lett. 73, 95-100. doi: 10.1016/0304-3940(87)90038-3

Ringstad, N., Abe, N., and Horvitz, H. R. (2009). Ligand-gated chloride channels are receptors for biogenic amines in C. elegans. Science 325, 96-100. doi: 10. 1126/science. 1169243

Sangaletti, R., and Bianchi, L. (2013). A method for culturing embryonic C. elegans cells. J. Vis. Exp. 79:e50649. doi: 10.3791/50649

Scemes, E., Suadicani, S. O., Dahl, G., and Spray, D. C. (2007). Connexin and pannexin mediated cell-cell communication. Neuron Glia Biol. 3, 199-208. doi: $10.1017 / \mathrm{s} 1740925 \times 08000069$

Shaham, S. (2005). Glia-neuron interactions in nervous system function and development. Curr. Top. Dev. Biol. 69, 39-66. doi: 10.1016/s0070-2153(05) 69003-5

Shaham, S. (2006). Glia-neuron interactions in the nervous system of Caenorhabditis elegans. Curr. Opin. Neurobiol. 16, 522-528. doi: 10.1016/j.conb.2006. 08.001

Shaham, S. (2010). Chemosensory organs as models of neuronal synapses. Nat. Rev. Neurosci. 11, 212-217. doi: 10.1038/nrn 2740

Shao, Z., Watanabe, S., Christensen, R., Jorgensen, E. M., and Colon-Ramos, D. A. (2013). Synapse location during growth depends on glia location. Cell 154, 337350. doi: 10.1016/j.cell.2013.06.028

Simonsen, K., Moerman, D., and Naus, C. C. (2014). Gap junctions in C. elegans. Front. Physiol. 5:40. doi: 10.3389/fphys.2014.00040

Smythies, J., and Edelstein, L. (2014). Telocytes, exosomes, gap junctions and the cytoskeleton: the makings of a primitive nervous system? Front. Cell. Neurosci. 7:278. doi: 10.3389/fncel.2013.00278

Spencer, W. C., Zeller, G., Watson, J. D., Henz, S. R., Watkins, K. L., McWhirter, R. D., et al. (2011). A spatial and temporal map of C. elegans gene expression. Genome Res. 21, 325-341. doi: 10.1101/gr.114595.110

Starich, T., Sheehan, M., Jadrich, J., and Shaw, J. (2001). Innexins in C. elegans. Cell Commun. Adhes. 8, 311-314. doi: 10.3109/15419060109080744

Stout, R. F. Jr., Grubisic, V., and Parpura, V. (2013). A Caenorhabditis elegans locomotion phenotype caused by transgenic repeats of the hlh-17 promoter sequence. PLoS One 8:e81771. doi: 10.1371/journal.pone.0081771

Stout, R. F. Jr., and Parpura, V. (2011). Voltage-gated calcium channel types in cultured C. elegans CEPsh glial cells. Cell Calcium 50, 98-108. doi: 10.1016/j. ceca.2011.05.016

Stout, R. F. Jr., and Parpura, V. (2012). Cell culturing of Caenorhabditis elegans glial cells for the assessment of cytosolic Ca2(+) dynamics. Methods Mol. Biol. 814, 153-174. doi: 10.1007/978-1-61779-452-0_12

Strange, K., Christensen, M., and Morrison, R. (2007). Primary culture of Caenorhabditis elegans developing embryo cells for electrophysiological, cell biological and molecular studies. Nat. Protoc. 2, 1003-1012. doi: 10.1038/nprot. 2007.143
Suadicani, S. O., Cherkas, P. S., Zuckerman, J., Smith, D. N., Spray, D. C., and Hanani, M. (2010). Bidirectional calcium signaling between satellite glial cells and neurons in cultured mouse trigeminal ganglia. Neuron Glia Biol. 6, 43-51. doi: $10.1017 / \mathrm{s} 1740925 \times 09990408$

Thomas, J. H. (1994). The mind of a worm. Science 264, 1698-1699. doi: 10. 1126/science.7911601

Verkhratsky, A., and Butt, A. M. (2013). Glial Physiology and Pathophysiology. Chichester, UK: John Wiley and Sons.

Voutev, R., and Hubbard, E. J. (2008). A "FLP-Out" system for controlled gene expression in Caenorhabditis elegans. Genetics 180, 103-119. doi: 10. 1534/genetics.108.090274

Vowels, J. J., and Thomas, J. H. (1994). Multiple chemosensory defects in daf-11 and daf-21 mutants of Caenorhabditis elegans. Genetics 138, 303 -316 .

Wadsworth, W. G., Bhatt, H., and Hedgecock, E. M. (1996). Neuroglia and pioneer neurons express UNC-6 to provide global and local netrin cues for guiding migrations in C. elegans. Neuron 16, 35-46. doi: 10.1016/s0896-6273(00) 80021-5

Wallace, R. L., and Smith, H. A. (2013). "Rotifera," in eLS. Chichester, UK: John Wiley and Sons, Ltd.

Wang, Y., Apicella, A. Jr., Lee, S. K., Ezcurra, M., Slone, R. D., Goldmit, M., et al. (2008). A glial DEG/ENaC channel functions with neuronal channel DEG-1 to mediate specific sensory functions in C. elegans. EMBO J. 27, 2388-2399. doi: 10.1038/emboj.2008.161

Wang, Y., D’Urso, G., and Bianchi, L. (2012). Knockout of glial channel ACD1 exacerbates sensory deficits in a C. elegans mutant by regulating calcium levels of sensory neurons. J. Neurophysiol. 107, 148-158. doi: 10.1152/jn.00299. 2011

Ward, S., Thomson, N., White, J. G., and Brenner, S. (1975). Electron microscopical reconstruction of the anterior sensory anatomy of the nematode Caenorhabditis elegans.?2UU. J. Comp. Neurol. 160, 313-337. doi: 10.1002/cne.90160 0305

White, J. G., Southgate, E., Thomson, J. N., and Brenner, S. (1986). The structure of the nervous system of the nematode Caenorhabditis elegans. Philos. Trans. R. Soc. Lond. B Biol. Sci. 314, 1-340. doi: 10.1098/rstb.1986. 0056

Yates, D. (2013). Neuronal circuits: staying connected during growth. Nat. Rev. Neurosci. 14, 588-589. doi: 10.1038/nrn3571

Yoshimura, S., Murray, J. I., Lu, Y., Waterston, R. H., and Shaham, S. (2008). mls-2 and vab-3 control glia development, hlh-17/Olig expression and glia-dependent neurite extension in C. elegans. Development 135, 2263-2275. doi: 10.1242/dev. 019547

Conflict of Interest Statement: The authors declare that the research was conducted in the absence of any commercial or financial relationships that could be construed as a potential conflict of interest.

Received: 13 January 2014; accepted: 15 February 2014; published online: 14 March 2014.

Citation: Stout RF Jr, Verkhratsky A and Parpura V (2014) Caenorhabditis elegans glia modulate neuronal activity and behavior. Front. Cell. Neurosci. 8:67. doi: 10.3389/fncel.2014.00067

This article was submitted to the journal Frontiers in Cellular Neuroscience.

Copyright (c) 2014 Stout, Verkhratsky and Parpura. This is an open-access article distributed under the terms of the Creative Commons Attribution License (CC BY). The use, distribution or reproduction in other forums is permitted, provided the original author(s) or licensor are credited and that the original publication in this journal is cited, in accordance with accepted academic practice. No use, distribution or reproduction is permitted which does not comply with these terms. 